\title{
THE THEORY OF HIGH ENERGY COLLISION PROCESSES
}

\author{
Principal Investigator: Tai Tsun Wu \\ School of Engineering and Applied Sciences \\ Harvard University \\ Cambridge, Massachusetts 02138
}

\author{
Final Report DOE/ER/40158-1 \\ U.S. Department of Energy \\ DOE Grant No. DE-FG02-84ER40158 \\ for the period \\ June 1, 1984 through November 30, 2007 \\ September 15, 2011
}

\begin{abstract}
NOTICE
This report was prepared as an account of work sponsored by the United States Government. Neither the United States nor the Department of Energy, nor any of their employees, nor any of their contractors, subcontractors, or their employees, makes any warranty, express or implied, or assumes any legal liability or responsibility for the accuracy, completeness, or usefulness of any information, apparatus, product or process disclosed, or represents that its use would not infringe privately-owned rights.
\end{abstract}

Prepared for

Contracts Management Office

United States Department of Energy

Chicago Operations Office

9800 South Cass Avenue

Argonne, Illinois 60439 


\begin{abstract}
Prior to 1984, The U.S. Department of Energy had supported Tai Tsun Wu's research at Harvard on the theory of high energy collision processes for many years. With this support from DOE, Professor Wu and his Ph.D. students, Post-Doctoral visitors, and distinguished collaborators had produced a large body of scientific advances in high-energy physics. These included a comprehensive theory for scattering processes at extremely high energies, based on relativisitic quantum field theories. A simple physical picture called the impact picture was obtained which gave a number of unusual predictions, many of which were verified experimentally. An especially accurate experimental verification of the prediction of increasing total cross section was provided by the CERN $p \bar{p}$ Collider at a center-of-mass energy of $540 \mathrm{GeV}$. This agreement was found to hold also with the data from the higher-energy Tevatron Collider at the Fermi National Accelerator Laboratory.

In 1984, DOE awarded Harvard University a new Grant DE-FG02-84ER40158 to continue their support of Tai Tsun Wu as Principal Investigator of research on the theory of high energy collision processes. This Grant was continued and renewed periodically and was in effect continuously until November 30, 2007. Topics of interest during the 23year duration of this Grant include: theory and phenomenology of collision and production processes at ever higher energies; helicity methods for QED and QCD; neutrino oscillations and masses; Yang-Mills gauge theory; Beamstrahlung; Fermi pseudopotentials; magnetic monopoles and dyons; cosmology and related topics; classical confinement; mass relations; Bose-Einstein condensation; and large-momentum-transfer scattering processes. This Final Report describes the research carried out on Grant DE-FG02-84ER40158 for the period June 1, 1984 through November 30, 2007. Two books resulted from this project and a total of 125 publications.

After the conclusion of this Grant, efforts were made to obtain theoretical predictions relevant to the experiments soon to be carried out at the Large Hadron Collider at CERN. This Large Hadron Collider is a proton-proton colliding accelerator designed for the center-of-mass energy of $14 \mathrm{TeV}$, much above that of the Tevatron Collider. A number of interesting results on production processes, again based on relativistic quantum field theories, have been obtained.
\end{abstract}




\section{A. INTRODUCTION}

The basic theme of this program is to extract information from relativistic quantum field theory, especially in the limit where some energy and/or momentum is much larger than the relevant masses. Physically, simplifications may be expected in this limit, an example being geometrical optics as compared with classical electromagnetic theory.

In the present program, gauge field theory in four dimensions has been studied from this point of view. In the case of elastic scattering, the most interesting limit considered is the one where both of the incident particles are extremely relativistic in the center-ofmass system, and this limit is studied through the perturbation series. Perhaps the most unexpected prediction of this approach by the Principal Investigator is that, in all cases, the total cross sections must increase without limit at very high energies. For the $p p$ and $\bar{p} p$ cases, we have also developed a phenomenology on the basis of this theory, and many of the resulting predictions, including not only the increasing total cross section but also the rising plateau and the increasing $\sigma_{\mathrm{el}} / \sigma_{\mathrm{tot}}$, have been accurately verified experimentally at the CERN ISR, the Fermilab fixed target, the CERN SPS, the CERN $p \bar{p}$ Collider, and the Fermilab Tevatron Collider. Very recently, we have generalized these predictions to the cases of $\pi^{+} p, \pi^{-} p, K^{+} p$, and $K^{-} p$ elastic scattering, but the predictions still need to be compared with data in the future.

When the transverse momenta are of the same order of magnitude as the total energy,

we have developed the helicity method for QED and QCD. Several dozens of such processes have been treated, and the results have been found to be useful by experimentalists.

Since gauge field theory is the basis of this development, there are efforts continuously by the Principal Investigator to study the Yang-Mills non-Abelian gauge field theory. An especially interesting case is the one where the gauge group is not semisimple. In these cases, we have found that there may be additional relevant gauge fields, called Yang-Mills fields of the second kind, which appear in the gauge transform of the original Yang-Mills fields. If such a theory can be developed, then the question will be raised whether the highenergy behavior of such a theory is similar or different from that of semisimple groups. 
A second topic for the theory of high energy collision processes is neutrino physics. As first shown conclusively by the Super-Kamiokande experiment, neutrinos are not massless. Thus the Standard Model of Glashow, Weinberg and Salam is modified slightly by the addition of three right-handed neutrinos.

The existing experimental data on neutrino oscillations give the values for the differences of the squares of the neutrino masses. In order to determine the individual neutrino masses, it is necessary to use these data together with suitable theoretical input. By using the similarity between quarks and leptons, we have found such a theoretical input in a natural way. We have studied the case of quarks and found on theoretical grounds a relation between quark masses and the Cabibbo-Kobayashi-Maskawa matrix, and this relation agrees well with experimental data. A generalized version of this relation was then applied to the case of leptons. In this way, there are only three unknown parameters for the leptons, namely, the three neutrino masses, since the masses of the charged leptons are accurately known. These three neutrino masses can then be determined by a $\chi^{2}$ fit to the solar and atmospheric data on neutrino oscillation. The fit to the solar data requires an understanding of the MSW effect for three neutrino species, as discussed above. This is necessary because the two-neutrino approximation is neither general enough nor accurate enough. In this way, we have determined the masses for the three neutrinos to be $2-5$, 10-13, and 52-54 meV (milli-electron-volts), respectively. 


\section{B. SUMMARY OF 23 YEARS OF RESEARCH AT HARVARD UNIVERSITY UNDER PRINCIPAL INVESTIGATOR TAI TSUN WU, WITH THE SUPPORT OF THE DEPARTMENT OF ENERGY UNDER GRANT DE-FG02-84ER40158}

\section{Theory and Phenomenology of Hadronic High-Energy Collision Processes}

Major collaborators: Hung Cheng (Professor of Mathematics, M.I.T.), and Claude Bourrely and Jacques Soffer (C.N.R.S., Marseille, France)

\section{a) Background and Overview}

Topic 1 is the central topic of the present research, as indicated by the title of this Grant, "The Theory of High Energy Collision Processes." In this Final Report, it seems an opportune occasion to summarize the work on this topic, including the work under the previous DOE Contract DE-AC02-76ER03227 with the same Principal Investigator. The purpose here is to study, both theoretically and phenomenologically, collision processes at high energies, including especially proton-proton and proton-antiproton collisions.

Just over fifty years ago, during the 1958 Annual International Conference on High Energy Physics (the Rochester Conference) held at CERN, Geneva, Switzerland, J. Robert Oppenheimer gave the Concluding Remarks. In his speech, Oppenheimer said, among other things, the following:

"There are areas where we know very little - extremely high energy collisions, for example - where little can be done by anyone."

Nearly ten years later, in 1967, a decision was reach by the Atomic Energy Commission, the predecessor of the Department of Energy, to fund the Fermi National Accelerator Laboratory (FNAL). The new accelerator was originally designed to be a $200-\mathrm{GeV}$ proton accelerator on a fixed target. This high energy of $200 \mathrm{GeV}$ makes it possible to study, for the first time, proton-proton interactions where both of the incident particles are extremely relativistic in the center-or-mass system. After learning about this decision, Tai Tsun Wu, the Principal Investigator of this Grant, together with Hung Cheng, decided that it was time to accept Oppenheimer's challenge to study extremely high energy collisions.

The Approach. At extremely high energies, the wavelengths of the incident hadrons are small, perhaps much smaller than the sizes of these hadrons. It is this property that may be expected to simplify the physical picture at such very high energies. This is analogous 
to our daily experience: our eyes function because geometric optics is much simpler than the Maxwell equations.

The problem is therefore to find a way to obtain an "optics-like" physical picture for hadronic collisions at extremely high energies. Of course, this physical picture is expected to be quite complicated, certainly much more complicated than optics.

Interactions between elementary particles share the following basic and important features:

- three spatial dimensions and one time dimension,

- relativistic kinematics,

- unitarity, and

- particle production.

Even though each of these properties is simple in nature, it is nevertheless not easy to formulate a model that incorporates all four features. The simplest way to have these features is to have a four-dimensional relativistic quantum field theory.

Choice of Relativistic Quantum Field Theory. In order to be able to make predictions for the high energy collision processes of hadrons, it is important to choose a proper relativistic quantum field theory. The choice was made in two steps.

For the first step, the 1954 paper of Yang and Mills is invoked: all interactions in physics should be described by gauge field theory, i.e., relativistic quantum field theory with a gauge invariance of the second kind. In other words, the choice should be restricted to gauge field theory.

There are two distinct types of gauge field theory:

- Abelian gauge field theory, and

- non-Abelian gauge field theory, or Yang-Mills theory.

Here, the term "Abelian gauge field theory" means a gauge field theory with a U(1) gauge group, i.e., a theory similar to quantum electrodynamics except that the vector gauge particle is not massless. On the other hand, the term "Yang-Mills theory" means a gauge field theory with a non-Abelian gauge group, the simplest example being the group SU(2).

The second step of the choice is to use the Abelian gauge theory to study the Oppenheimer problem of hadronic collisions at extremely high energies. This choice requires a discussion, because it is known that the strong interactions of hadrons are not described by an Abelian gauge theory, but rather by quantum chromodynamics, i.e., a Yang-Mills theory with the non-Abelian gauge group $\mathrm{SU}(3)$. 
Strictly speaking, this second step of the choice is not a choice at all, i.e., it is forced upon us. Unlike optics, the determination of the high-energy behavior for the Abelian gauge theory is by no means simple - see below. In the seventies and eighties, there was a great deal of effort, by many theoretical physicists, to try to determine the high-energy behavior of the simplest Yang-Mills theory, i.e., the SU(2) non-Abelian gauge theory. It is now known that most of these efforts have not worked out. In other words, even more than forty years after the funding of the FNAL accelerator in 1967, essentially nothing is known about the high-energy behavior of the SU(2) Yang-Mills non-Abelian gauge theory.

Under these circumstances, it was a fortunate decision in the sixties to concentrate on the study of the high-energy behavior of the four-dimensional Abelian gauge theory.

Summation of Leading Terms. After the choice is made to study the high-energy behavior of the Abelian gauge theory, the question is how this high-energy behavior can be determined. For this purpose, a major assumption has to be introduced.

No four-dimensional gauge theory has ever been solved exactly. As a consequence, the main handle for such a theory is its perturbative expansion. In order to extract its high-energy behavior, it is necessary to study the perturbation series to all orders.

A high-order term in this perturbation series is extremely complicated, being given by the sum of contributions from many Feynman diagrams. In order to get something manageable, it is essential to look for simplifications for high energies.

In the simplest gauge theory, there are a fermion and a vector gauge particle. Let their masses be taken to be of the same order of magnitude, say $m$. For two-body elastic scattering near the forward direction, there is one large parameter, namely,

$$
\frac{\sqrt{s}}{m}
$$

where $\sqrt{s}$ is the center-of-mass energy. A term of a given order in the perturbation series can then be evaluated asymptotically for large values of this parameter. The leading term of this asymptotic expansion for a given order is much simpler than the original expression, many of the Feynman diagrams not contributing. These leading terms are then summed up.

While there is no rigorous mathematical reason why this sum of leading terms must give the desired high-energy behavior, there are many examples from other branches of physics where such a sum of leading terms gives the correct answer. On that basis, it is assumed that, for the problem of high-energy collision processes, this sum does give the desired answer. 
At this point, it is instructive to compare the Abelian case with the SU(2) Yang-Mills non-Abelian gauge theory. For the specific case of fermion-fermion elastic scattering in the nearly forward direction, the present situation is as follows:

i) For the Abelian case, the leading terms are known to all orders, but

ii) For the SU(2) Yang-Mills non-Abelian gauge theory, the leading term is known for the sixth order, but not known even for the eighth order. (For the tenth order, it is not even known which Feynman diagrams contribute to the leading order.)

This demonstrates explicitly the fortunate choice mentioned above.

Elastic Scattering - Theory and Phenomenology. The approach described above was applied to elastic scattering. The first surprising result was that the total cross section for two-body scattering increases without bound for higher and higher energies. Since this result was totally unexpected, it was desirable to have experimental verification. The experimentalists who were interested in carrying out the measurements raised a most relevant and proper question: Could we give them an estimate of the amount of increase in total cross section, so that they could plan their experiments?

This led to the development of a phenomenology. This was developed by the Principal Investigator, Tai Tsun Wu, in collaboration with Claude Bourrely and Jacques Soffer, both of CNRS-Luminy of France. Much of this work on elastic scattering can be found in:

Hung Cheng and Tai Tsun Wu, Expanding Protons: Scattering at High Energies, The MIT Press, Cambridge, MA, 1987.

Tai Tsun Wu, "Scattering and Production Processes at High Energies," in Scattering-Scattering and Inverse Scattering in Pure and Applied Science, Vol. 2, R. Pike and P. Sabatier, Eds., pp. 1582-1604, Academic Press, London, 2001.

It is perhaps worth emphasizing that the present work is very much motivated by proposed experiments. It started a few years before the construction of the 200-GeV proton accelerator at the Fermi National Accelerator Laboratory, and ended a few years before the shut down of the Tevatron Collider in 2011 at the same Laboratory.

Production of Higgs Particle. The term "High Energy Collision Process" includes not only elastic scattering but also production processes. The work described above has been mostly on elastic scattering, the reason being the lack of prospective experimental data on interesting production processes. With the operation of the Large Hadron Collider (LHC) 
at CERN, this situation has changed dramatically: the production of the Higgs particle in proton-proton collisions is clearly a new and important phenomenon.

The work on this production process has occurred after the end of the present Grant. Nevertheless, for completeness it is worthwhile to discuss the theory of Higgs production with the same approach.

At LHC, the Higgs particle can be produced by various reactions, one of the important ones being

$$
p+p \rightarrow p+H+p,
$$

where the Higgs particle $H$ has a relatively low energy in the center-of-mass system. For these production processes, there are two large parameters, namely,

$$
\frac{\sqrt{s}}{M} \quad \text { and } \quad \frac{M}{n},
$$

where $M$ is the Higgs mass.

For the study of this Higgs production process, the task is to re-develop, or shall we say generalize, the original theory developed on this Grant for the asymptotic analysis of Feynman amplitudes with one large parameter to that with two large parameters, together with a phenomenological model to give an accurate description of this process. Both of these have been accomplished recently (Nucl. Phys. B 850, 53, 2011).

The next step is to calculate the inclusive Higgs production cross section

$$
p+p \rightarrow H+\text { anything. }
$$

It is not clear how this can be carried out with the present approach, but it is important to accomplish this.

Only after this and related production processes are understood theoretically and verified experimentally, can it be said honestly that the 1958 challenge of Oppenheimer has been met more than fifty years later.

\section{b) Details of Research Accomplished}

With the background and overview given in section 1a) above, the specific activity on this project on high-energy collision processes is summarized below. This project has been carried out successfully for many years under DOE support. During the previous DOE Grant DE-AC02-76ER03227 [1], a total of 75 papers on this topic were published during a period of many relevant high-energy experiments. The research led to many theoretical results that were later confirmed experimentally. 
In 1984, just prior to the beginning of this DOE Grant DE-FG02-84ER40158, data were collected from the CERN $p \bar{p}$ Collider at $\sqrt{s}=0.546 \mathrm{TeV}$ that, again, confirmed the predictions of our "impact picture" which was based on quantum field theory. Soon after, Bourrely, Soffer and I extended these predictions on $p p$ and $p \bar{p}$ total and elastic differential cross sections to higher energies up to $\sqrt{s}=40 \mathrm{TeV}$ - that were soon to be available at the CERN $p p$ collider and the Fermilab Tevatron collider. A number of interesting results were evident from this study, which was published in 1985 as Paper [7]. Perhaps the most obvious one was concerned with the possible characterization of the rapidly increasing inelastic cross section. It was presumed that both diffractive and pionization processes were involved.

Around the same time, an experiment was proposed for the measurement of Coulomb interference at the CERN Collider. Bourrely, Soffer and I elected to apply the "impact picture" to the Coulomb interference region for $p \bar{p}$ elastic scattering in the energy range of the CERN collider $(\sqrt{s}=546 \mathrm{GeV})$ and of the Fermilab Tevatron $(\sqrt{s}=1.8 \mathrm{TeV})$. The resulting predictions for the future experiments were published in 1987 as Paper [28].

Since the experimental data from the CERN $p \bar{p}$ Collider continued to confirm our predictions, Hung Cheng and I decided to present our theory in a coherent fashion in book form. The result, entitled "Expanding Protons: Scattering at High Energies" was published by M.I.T. Press in 1987 [2].

In 1988, Bourrely, Soffer and I published Paper [31], the purpose of which was threefold: 1) Since the Tevatron collider at Fermilab was ready to take more data, the first purpose was to present detailed predictions of our approach for the center-of-mass energy range of 0.8 to $2.0 \mathrm{TeV}$. 2) Because of the recent data at the CERN collider at $0.55 \mathrm{TeV}$ in the Coulomb interference region, our 1987 prediction [28] was compared with these data. 3) Lastly, since it seemed quite likely that the large hadron collider (LHC) and/or the superconducting super collider (SSC) would be built, the predictions of our approach were also given in some detail for two selected energies in the multi-TeV energy range. The paper presents three tables of theoretical predictions at the different energies, and four figures comparing our predictions to the recent experimental data.

Two years later, Paper [56] was published to compare the predictions in Paper [31] with experimental results from FNAL at the center-of-mass energy of $1.8 \mathrm{TeV}$. The experimental data compared very well with our predictions. At this energy, the theoretical predictions for $\sigma_{\text {tot }}$ and $\sigma_{\text {el }} / \sigma_{\text {tot }}$ were $74.8 \mathrm{mb}$ and 0.230 while the experimental results were $72.1 \pm 3.3$ $\mathrm{mb}$ and $0.23 \pm 0.02$, respectively. The data also confirmed the predictions for the elastic differential cross section. This is to be contrasted with the discrepancy on the ratio of 
the real and imaginary parts of the forward $p \bar{p}$ elastic scattering amplitude between the theoretical prediction and the data from the CERN $p \bar{p}$ Collider. For this reason, CERN decided to devote about six weeks of running time to repeat this measurement in November and December of 1991. In anticipation of this additional data, a more detailed study of the real part of the amplitude was carried out and published in 1991 as Paper [61]. A review of $p p$ and $p \bar{p}$ elastic scattering was also published in 1991 (see Paper [60]).

An important test of any reliable theoretical picture is its ability to describe the differential cross section $d \sigma / d t$ near the forward direction, more precisely in the Coulomb interference region where it is very sensitive to the real part of the hadronic amplitude. In 1993, theoretical predictions of the $p \bar{p}$ elastic scattering cross section were compared in Paper [83] with new UA4 data in the Coulomb interference region at the center-of-mass energy of $541 \mathrm{GeV}$. The agreement was excellent. A plot of the ratio of the experimental data to the theoretical prediction shows an oscillation of about (2-3)\%. There was no obvious explanation for this small oscillation. In the absence of an explanation, further experimental studies were needed to ascertain whether this oscillation was actually present.

The above developments all deal with hadron-hadron cross sections. It was realized from the early seventies that for many purposes the photon behaves like a hadron, but the phenomenology was not pursued at that time because there was no expectation of experimental measurements of $\gamma p$ cross sections at high energies. This situation changed in the early 1990s with the operation of the electron-proton colliding accelerator HERA at DESY, and it became desirable to have a prediction of photon-proton cross sections at high energies. In Paper [88], Bourrely, Soffer and I presented a very simple approach to this increasing $\gamma p$ total cross section, by expressing the $\gamma p$ cross section in terms of $\pi^{+} p$ and $\pi^{-} p$ cross sections. We showed that the rising total photoproduction cross section observed at HERA is entirely consistent with the impact picture in the theory of expanding protons. However, since the photon couples to all charged particles, there should be, in addition, contributions from the quarks of the second and the third generations. During the summer of $1995, \mathrm{Wu}$ was invited to give a review of the subject of rising cross sections at the Blois Workshop on "Frontiers in Strong Interactions," (Paper [95]), and a review of the more general subject of scattering by spherical objects at the 2nd Journées Maxwell at Bordeaux, France (Paper [94]). In both of these papers, the point is made that our prediction in Paper [88] should be modified to include the contributions from quarks of the second and the third generations to the $\gamma p$ cross section. These additional contributions can be disentangled experimentally by studying the $K$-meson contents of the $\gamma p$ total cross section. 
In view of the success with the increase in the total cross sections of $p p, p \bar{p}$, and $\gamma p$ scattering, the same was expected of the $\gamma \gamma$ total cross section. In 1997-98, two collaborations at LEP (L3 and OPAL) obtained data on this process, but these two sets of data were not quite consistent with each other. Analysis of the total cross section for photon-photon scattering at high energies was carried out by Bourrely, Soffer and myself and presented in Paper [106] in 2000. Both sets of data were found to be in rough agreement with the theoretical expectation. We had therefore encountered the expanding proton and photon in three distinct experimental situations: first the rising total cross section of $p p$ and $p \bar{p}$; secondly that of $\gamma p$ in 1994, and in 2000 that of $\gamma \gamma$. In Paper [113], Wu describes the present view of the theory of scattering at high energies, some recent new results, and answers to questions often asked. This was published in 2002 by Academic Press as Chapter 4.3.4 in the 2-volume book Scattering-Scattering and Inverse Scattering in Pure and Applied Science.

The next development was to apply the "impact picture" to $\pi^{+} p, \pi^{-} p, K^{+} p$, and $K^{-} p$ scattering at high energies. Unlike $p p$ scattering, there were no experimental data for pion-proton and kaon-proton scattering at sufficiently high energies so that the incident particles are extremely relativistic in their center-of-mass system. For this reason, the development of a phenomenology for these scattering processes was more difficult. It was accomplished in 2003 with the publication of Paper [119]. This paper gives the first successful generalization, leading to a phenomenology for these scatterings on a par with the previous $p p$ and $\bar{p} p$ cases. Although the new parameters presented in Paper [119] differ only slightly from the earlier ones obtained in 1984, they give significantly superior results for some of the differential cross sections. Furthermore, the present treatment of $\pi^{ \pm} p$ and $K^{ \pm} p$ elastic scattering may make it possible to deal with a number of additional scattering processes at high energies, the most interesting one being the inelastic diffraction $p+p \longrightarrow p+N(1440)$. This and a number of similar processes can perhaps be studied experimentally at RHIC at the Brookhaven National Laboratory.

Since the real part of the forward proton-proton scattering amplitude was never measured with any accuracy at the Fermilab Tevatron Collider, it was of particular interest to have the real part measured at the new Large Hadron Collider (LHC) at CERN. If the total cross section, as predicted by our expanding-proton theory, is verified by measurements at LHC, as expected, the question for theoreticians becomes, "Is it conceivable that the real part of the proton-proton forward scattering amplitude is different from the prediction of the same theory?" The answer is that this would require quite drastic modification of quantum field theory as we know it now. The simplest example is that the polynomial 
bounds are violated. We do not expect this to be the case; however, if the measurement gives significant deviation from the theoretical prediction, then there must be exciting and unexpected new physics. Two papers were published on this issue in 2006. Paper [123] was presented by $\mathrm{Wu}$ at the XIth Blois Conference in May, 2005; while Paper [124] is an abbreviated version that was published in a volume in memory of V. N. Gribov.

The measurement of spin observables in hadronic exclusive processes is the only way to obtain full knowledge of the corresponding set of scattering amplitudes, and, in particular, their relative size and phase difference. Taking the specific case of proton-proton elastic scattering, a reconstruction of the five amplitudes has been worked out in the low-energy domain in Paper [125]. This situation is very different at high energy; due to the lack of data, in the range $p_{\text {lab }} \simeq 100-300 \mathrm{GeV}$, besides the nonflip hadronic amplitude $\phi_{1}^{h}$, only the hadronic helicity-flip amplitude $\phi_{5}^{h}$ is known and to a rather poor level of accuracy. The advent of the Brookhaven National Laboratory Relativistic Heavy Ions Collider (BNL-RHIC) $p p$ collider, where the two proton beams can be polarized, longitudinally and transversely, up to an energy $\sqrt{s}=500 \mathrm{GeV}$, offers a unique opportunity to measure singleand double-spin observables, and thus to provide a determination of the spin-dependent amplitudes, which remain unknown so far.

In the framework of the impact picture we computed the analyzing power $A_{N}$ for $p p$ elastic scattering at high energy and in the very forward direction. We considered the full set of Coulomb amplitudes and showed that the interference between the hadronic nonflip amplitude and the single-flip Coulomb amplitude is sufficient to obtain a good agreement with the present experimental data. This led us to conclude that the single-flip hadronic amplitude is small in this low-momentum transfer region and it strongly suggests that this process can be used as an absolute polarimeter at the Relativistic Heavy Ions Collider, at Brookhaven National Laboratory, working as a polarized $p p$ collider.

A total of 19 publications on this subject were published between 1984 and 2007. They are Book [2] and Papers [7], [28], [30], [31], [56], [60], [61], [83], [88], [91], [94], [95], [106], [113], [119], and [123-125]. 


\section{Helicity Methods for QED and QCD}

Major collaborator: Raymond Gastmans (Professor of Theoretical Physics, University of Leuven, Leuven, Belgium)

Most of the work on this project can be found in Book [3]:

Raymond Gastmans and Tai Tsun Wu, The Ubiquitous PhotonHelicity Methods for QED and QCD, Oxford University Press, 1990.

Because of the book's availability, the description of the project will be relatively brief.

The rising total cross sections described above under Topic 1 are consequences of the high-energy behavior of scattering amplitudes for fixed transverse momentum transfers. After the completion of the theoretical derivation and the initial experimental confirmation of the rising cross sections, it was natural to raise the question as to what can be said about processes involving large transverse momentum transfers.

Under a lucky set of circumstances described in the Preface of the book mentioned above, Gastmans and Wu (Principal Investigator) embarked on the study of various processes where the transverse momentum transfers and the energies are both large and of the same order of magnitude. It was first realized that the asymptotic results on the cross sections for this limit are always unexpectedly simple, even though the intermediate steps in the derivations contain very complicated formulas. It took a few years of concentrated efforts to find a method of obtaining these simple results without getting involved in the complicated intermediate steps. This is the helicity method.

After this method was properly understood, we applied it to several dozens of processes in quantum electrodynamics and quantum chromodynamics. After a number of papers were written on both the method and its applications, we got tired of producing so many papers and then we decided to write a book instead. Consequently the book contains many results that are not available in the literature otherwise. Because of their simplicity, many of the results on cross sections and matrix elements have been used by experimentalists, including those working on LEP.

Eight publications on this subject were published in 1986-1990. They are Book [3] and Papers [15], [16], [22], [26], [33], [36], and [41]. 


\section{Neutrino Oscillations and Masses}

Major collaborators: Per Osland (Research Fellow and currently Professor at the

University of Bergen, Bergen, Norway),

Conrad Newton (Student and Research Fellow),

Harry Lehmann (Professor of Theoretical Physics, Hamburg University, Hamburg, Germany), and

Raymond Gastmans (Professor of Theoretical Physics, University of Leuven, Leuven, Belgium)

The Principal Investigator initiated this project and was joined by the four collaborators.

The only necessary modification to the very successful Standard Model of Glashow, Weinberg, and Salam as originally proposed is the introduction of neutrino masses, as required by the pioneering Super-Kamiokande experiment. This is easily accomplished by adding three right-handed neutrinos. In this way, there are three massive neutrinos, one for each generation.

\section{a) MSW Effects for Two Specific Electron Densities [Papers 111 and 107]}

Besides constant density, there are several cases of analytic electron densities, where the MSW equation for three-neutrino oscillation can be solved explicitly.

Linear density. In the case of two-neutrino oscillation, it is well known that the neutrino wave functions for linear electron density can be expressed in terms of the confluent hypergeometric function. In Paper [111], an alternative procedure has been found to solve this problem, and this alternative procedure has the important advantage of having a straightforward generalization to $N$-neutrino oscillation including, of course, $N=3$.

Exponential density. Again in the case of two-neutrino oscillation, the neutrino wave functions for exponential electron density can also be expressed in terms of the confluent hypergeometric function ${ }_{1} F_{1}$. This similarity does not carry over to the case of three neutrino species. Instead, in this case of exponential electron density, the ${ }_{1} F_{1}$ is replaced by the generalized hypergeometric function ${ }_{2} F_{2}$. In Paper [107], this ${ }_{2} F_{2}$ is studied in some detail since it is not a familiar function.

This case of exponential electron density gives a good approximation to that of neutrinos from the sun. Therefore, this is physically a more useful case in the understanding of neutrino oscillation.

Actually, besides these linear and exponential electron densities, there is a third case where the MSW equation for three-neutrino oscillation can be solved in terms of known functions. This case is more difficult mathematically. 


\section{b) Neutrino Masses [Papers 110, 112 and 118]}

Neutrino-oscillation experiments, such as those of Super-Kamiokande, SNO, etc., give the values for the differences of the squares of the neutrino masses. It is the purpose of this part of the project to determine what the individual neutrino masses are. These experiments do not give sufficient information by themselves to extract these mass values. The problem is therefore to find these individual masses by using the experimental data together with suitable theoretical inputs.

These theoretical inputs come from studying the related problem of the quark masses given in Paper [97]. Since symmetry considerations have played a central role in the development of particle physics, the idea here is to apply also symmetry considerations to the relations between the various parameters of the Standard Model. Specifically, it is postulated in Paper [97] that, in the so-called coherent basis, the contributions of the first-generation quarks to the quark mass matrices transform as the antisymmetric representations of two different subgroups [both isomorphic to the permutation group S(3)] of $S_{L}(3) \times S_{R}(3)$. It is then found that the mass matrices are completely determined by the quark masses, and the resulting CKM matrix is consistent with the available experimental data.

In the Standard Model, especially after the neutrinos are found to be not massless, there is a great deal of similarity between the properties of the six quarks and six leptons. It is therefore natural for us to apply the above results on the quarks also to the leptons. In this way, the lepton CKM matrix is expressed in terms of the masses of the six leptons. Since the masses of the three charged leptons are accurately known, there remain as unknown parameters only the masses of the three neutrinos.

This generalization from quarks to leptons is far from being trivial. One of the major difficulties is the following. Both for the three up quarks and the three down quarks, the masses are very different from each other; since the neutrino masses are not known, it is not proper to assume that this is also the case for the three neutrinos. Another complication is that it is not known whether $\mathrm{CP}$ is conserved or not in the lepton sector. Therefore, in addition to the possibility of "maximum" CP non-conservation, the case with $\mathrm{CP}$ conservation also has to be allowed.

Once the generalization to leptons is accomplished, the expected results for various experiments can be calculated as functions of the masses of the three neutrinos. For this step, an understanding of the Mikheyev-Smirnov-Wolfenstein effect for three neutrino species is essential. These results are then compared with the experimental data from 
Super-Kamiokande, Chlorine, SAGE, GALLEX, SNO, and CHOOZ. The three neutrino masses are determined using a $\chi^{2}$ fit.

The result we have found is that the mass of the lightest neutrino is $(2-5) \times 10^{-3} \mathrm{eV}$, that of the next heavier neutrino is $(10-13) \times 10^{-3} \mathrm{eV}$, while the mass of the heaviest neutrino is $(52-54) \times 10^{-3} \mathrm{eV}$.

Five publications on this subject were published in 2000-2003. They are Papers [107], [110]-[112], and [118].

\section{Yang-Mills Gauge Theory}

Major collaborator: Jean Nuyts (Professor of Theoretical Physics, University of Mons-Hainaut, Mons, Belgium)

In all applications of gauge field theory in four dimensions to physics, the gauge group is semisimple, possibly multiplied by one or more U(1)'s. In such cases, there are the following two remarkable properties. First, the number of gauge fields is equal to the number of generators for the gauge group. Secondly, given a gauge group, there is precisely one gauge field theory. It is natural to raise the question whether these properties hold for all gauge groups. Physically, this question may be important because we need to be alerted to such a possibility and, if a gauge group shows up without these properties, the expected results may be quite different.

In Paper [118] it was found that, for any gauge group, there is always a gauge field theory with the two properties mentioned above. If the gauge group is semisimple, then this is the only theory. However, if it is not semisimple, it is often possible to get further gauge field theories by introducing additional gauge fields, which we call Yang-Mills fields of the second kind. These Yang-Mills gauge fields of the second kind cannot be removed, because they enter into the gauge transformation of the original Yang-Mills fields. In such cases, of course neither of the two properties hold. It remains to make sense of these Yang-Mills gauge fields of the second kind.

Four publications on this subject were published during 1987-2003. They are Papers [20], [31], [115], and [118]. 


\section{Beamstrahlung}

Major Collaborator: Maurice Jacob (CERN, Geneva, Switzerland)

It has been known for some time that, in order to reach $e^{+} e^{-}$center-of-mass energies much above the $200 \mathrm{GeV}$ already achieved at LEP, there is no alternative but to build electron-positron linear colliders. The reason is that circular colliders unavoidably give off too much synchrotron radiation.

The single-pass nature of electron-positron linear colliders imposes strong constraints on the design of such accelerators. In particular, useful luminosities can be reached only by making the radii of the bunches exceedingly small. One of the consequences of such tight bunches is that there is significant radiation when the opposing bunches penetrate each other. This radiation is called beamstrahlung.

About 1985, three groups began to study beamstrahlung almost simultaneously, the other two being Blankenbecler and Drell at SLAC and Bell and Bell at CERN. Our approach differs from those of the other two groups mainly in the use of Feynman diagrams ab initio. The nature of the necessary approximations and possible improvements has been summarized in the book by Gastmans and $\mathrm{Wu}[3]$ mentioned under Topic 2 above.

As a first step, we introduced a new correlation length that is appropriate to beamstrahlung. We then concentrated on the case where this correlation length is much less than the bunch length, which is in turn much less than the so-called virtual electron length. This ordering of the lengths is expected to hold for multi-TeV electron-positron linear colliders. A number of cases have been treated, including single-photon beamstrahlung for bunches that form uniform circular cylinders and for longitudinally non-uniform bunches, and also multi-photon beamstrahlung from uniform bunches. One of the especially difficult problems that has been studied is the treatment of transversely non-uniform bunches. A typical case has been solved, but the practically important case of bunches that are non-uniform both longitudinally and transversely remains to be treated.

A total of 14 publications have been published on this subject during 1987-1993. They are Papers [29], [32], [35], [37]-[40], [42], [43], [68]-[70], [73], and [80]. 


\section{Fermi Pseudopotentials}

Major Collaborators: Alexander Grossmann (Centre de Physique II, CNRS, Marseille, France), and D. Kent Freeman (Student)

Under the previous DOE Grant [1], Fermi pseudopotentials were defined in a useful way in higher dimensions by A. Grossmann and T. T. Wu [J. Math. Phys. 25, 1742 (1984)], despite a well-known mathematical theorem that this is possible only in three or fewer dimensions. This contradiction was traced to the choice of the underlying space. In Paper [6] of the present Grant, the use of Pontryagin spaces was found to avoid such limitations.

This generalization opened up the study of large classes of problems, including those involving Maxwell's equations. In particular, an array of pseudopotentials can be arranged such that there are extremely narrow resonances, as described in Paper [27]. Student, D. Kent Freeman, wrote his doctoral dissertation on such arrays, the title of his thesis being "Extremely Narrow Resonances in Closed-Loop Arrays of Quantum Mechanical and Electromagnetic Interactions." Freeman's research also led to a series of papers by him: Papers [65], [72], [87]. Paper [90] was the result a collaboration of Freeman and Wu on a variational-principle formulation of the two-term theory for arrays of cylindrical dipoles.

Six publications have been published on this subject between 1984 and 1995. They are Papers [6], [27], [65], [72], [87], and [90].

\section{Magnetic Monopoles and Dyons}

Major collaborator: Per Osland (Research Fellow and currently Professor at the University of Bergen, Norway)

In 1975, T. T. Wu and C. N. Yang [Phys. Rev. D 12, 3845 (1975)] realized that the magnetic monopole is naturally described using fibre bundles. This work was carried out under the previous DOE Contract DE-AC02-76ER03227 [1]. With this set up using fibre bundles, a number of problems involving magnetic monopoles and dyons were studied in detail.

Per Osland and I began a collaboration in 1984 on monopole-fermion and dyon-fermion bound states that resulted in a series of six papers I-VI. In Paper I [4], we investigated the general case of dyon-fermion bound states through series expansions on the basis of the hamiltonian. These series expansions were used to obtain highly accurate numerical results, which gave an overview of the properties of the bound states and were also used to ascertain the accuracy of the approximate results developed in Papers II-VI, in which two limiting cases were investigated and the eigenvalues and wave functions were determined 
approximately by analytic methods. Papers II [5], III [11] and IV [12] deal with the case of the lowest angular momentum $j=|q|-\frac{1}{2}$. For this case, the wave functions do not depend on $\kappa$ and $|q|$ separately, but only on the product $\kappa|q|$. For these states, the two limiting cases studied are those of weak binding (in Paper II) and of large values of $\kappa|q|$ (in Papers III and IV). The treatment of weak binding is then extended in Papers V [13] and VI [14] to higher angular-momentum states.

Nine publications on this subject have been published between 1984 and 1990. They are Papers [4], [5], [11]-[14], [19], [20], and [52].

\section{Cosmology}

Scott Dodelson (Research Fellow) studied a number of problems of interest to cosmology. He invented a number of scenarios - some with new postulated particles - for various possibilities in cosmology including, in particular, baryogenesis and properties of dark matter.

A total of 10 papers have been published on this subject between 1989 and 1992. They are Papers [44], [45], [49], [51], [57], [62]-[64], [78], and [79].

\section{Noncommutative Geometry}

Robert Coquereaux (Research Fellow) has a very broad range of interests. He is the first person to define, in the context of the Standard Model, an effective charge that is renormalization-scheme independent. Using noncommutative geometry, he has studied possible relations between the masses of the fermions and the boson in the Standard Model.

Not counting those published with support from the previous DOE Contract DE-AC0276ER03227, 4 papers have been published on this subject in 1989 and 1990. They are Papers [46]-[48], and [58].

\section{Classical Confinement and Slowly Decaying Electromagnetic Pulses}

Major collaborator: Harry Lehmann (Professor of Theoretical Physics, Hamburg University, Hamburg, Germany)

Research on classical models of confinement was initiated near the end of the previous DOE Grant and resulted in Paper I by Lehmann and Wu published in 1984 [1]. Lehmann and I continued to work on classical confinement under the new DOE Grant beginning in June 1984. The results of our efforts were published in Papers [8] and [17]. In particu- 
lar, in Paper [17] we found that our previous treatment could be generalized to uniformly moving quark and anti-quark sources. The more fundamental problem was to inquire how confinement arises from QCD. In Paper [8], the approach was to study SU(2) gauge theory on a small lattice. It was found that in this case the classical trajectories have nearby kinetic foci, which is qualitatively different from the corresponding Abelian case.

In 1983, J.N. Brittingham proposed the problem of searching for classical electromagnetic waves with the properties of (i) satisfying the homogeneous Maxwell's equations, (ii) being continuous and nonsingular, (iii) having a three-dimensional pulse structure, (iv) being nondispersive for all time, (v) moving at light velocity in straight lines, and (vi) carrying finite electromagnetic energy. This "focus wave mode" of Brittingham contained infinite energy, and it was much discussed whether finite-energy solutions of a similar nature exist. Such solutions, if they were to exist, would be of considerable theoretical interest and practical importance. Unfortunately, the explicit example given by Brittingham turned out not to satisfy property (i), and it was generally believed that no such electromagnetic waves could exist.

In 1985 Lehmann and I attempted to set up a framework for the investigation of problems of this type. This work was facilitated greatly by the intuition gained from concurrent advances in particle physics. In particular, it was learned from this work that, even for extremely short wavelengths, logarithmic dependence of various quantities such as the total cross section on the wavelength can, and does, occur. Papers [9] and [10] were published on this subject in 1985. In Paper [9], a specific formulation was proposed. On the basis of this formulation, it was found that, for a given "detector," the energy received by the detector must approach zero as the detector is moved further and further away from the antenna. However, this approach to zero need not be of the form $R^{-2}$, but can instead be much slower. An electromagnetic pulse with such slow decay was conveniently referred to as an "electromagnetic missile." Some simple properties and examples of electromagnetic missiles were given.

In Paper [10] it was shown that for any finite-energy solution of the free-space Maxwell equations without sources, the energy contained in an infinite slab of finite thickness approaches zero as time tends to infinity. When applied to the work of Brittingham, the result was that the fraction of energy in the focal region is infinitesimal, so that the "focus wave mode" does not focus.

Four publications on these subjects were published in 1985 and 1986. They are Papers [8]-[10], and [17]. 


\section{Mass Relations}

Major collaborators: Harry Lehmann (Professor of Theoretical Physics, Hamburg University, Hamburg, Germany),

Conrad Newton (Student),

Per Osland (Research Fellow and currently Professor at the University of Bergen, Bergen, Norway)

Most of this work involves attempts to find mass relations by assuming the absence of some divergence. This attempt, involving Papers [74]-[77], [82], [84], and [87], has not been successful.

The only successful part of this work is that on the quark mass matrices in Paper [92]. This result has been used to determine the neutrino masses (see Topic 3 above). The neutrino masses determined this way are in good agreement with those obtained from other considerations.

\section{Coupled Channels}

Major collaborators: N. N. Khuri (Rockefeller University, New York, NY), and André Martin (CERN, Geneva, Switzerland)

A number of questions have been studied in the context of non-relativistic problems. In particular, new singularities have been found and stability regions have been studied.

A total of 12 papers have been published on this subject between 1992 and 2006. They are Papers [71], [81], [93], [99], [100], [103], [104], [108], [114], [115], [121], and [122].

\section{Bose-Einstein Condensation}

Major collaborator: Dionisios Margetis (Student and Research Fellow)

In 1995, in a remarkable paper, Anderson, Ensher, Matthews, Wieman and Cornell reported the first experimental observation of Bose-Einstein condensation (BEC) in a weakly interacting system. Many experiments on various atomic vapors followed rapidly.

From the theoretical point of view, the treatment of this problem involves non-perturbative effects in quantum field theory. In particular, an important new feature of these experiments is the presence of a trap which is needed to keep the atoms together.

With or without this external potential that describes the trap, it has been found that the minimum description of BEC involves a condensate wave function and the pair excitation function, the latter being responsible for the existence of phonons. The equations of 
motions for these functions have been found for very low temperatures. The experimentally interesting case of a slowing varying external potential has been studied in detail; soliton solutions have also been found.

A total of 3 papers have been published on this subject in 1997-1999. They are Papers numbered [98], [102], and [105].

\section{High-Energy Large-Momentum-Transfer Scattering Processes}

Major collaborators: Conrad Newton (Student and Research Fellow), and

Per Osland (Research Fellow and currently Professor at the University of Bergen, Bergen, Norway)

After studying the rising cross sections, as discussed under Topic 1, attention was turned to the case where the transverse momentum transfer is of the same order of magnitude as the energy, which is described under Topic 2. It was soon realized that these two cases are the two extremes of high-energy scattering processes: $t$ is fixed in the former case and $t / s$ is fixed in the latter case. There is a vast region in between where $s$ is much larger than $|t|$, which is in turn much larger than the squares of the masses involved. It is the purpose of this project to fill this large gap.

We began by studying the scalar case of $\phi^{3}$ theory. Indeed, this is the case that started the investigation of high-energy behavior of quantum field theory, one of the very early results being the appearance of Regge poles. Several years ago, Osland and Wu obtained the asymptotic behaviors, for high energies and large momentum transfers, of the one-, two-, and three-loop ladder diagrams in $\phi^{3}$ theory. In each case, the asymptotic results are fairly simple, but not simple enough for us to guess the situation in the more general case of $n$ loops. Furthermore, the method used was not powerful enough to deal with four loops.

Conrad Newton decided that he wanted to write his doctoral dissertation on this general $n$-loop case. He performed the remarkable feat of solving this problem completely. Of course this work became his thesis. Later, together with Raymond Gastmans and Walter Troost of the University of Leuven, two related cases in $\phi^{3}$ theory have also been studied. Neither of these cases is anywhere near as difficult as the one solved by Newton. Newton contributed to Topic 3, as already discussed above.

A total of 4 papers have been published on this subject between 1987 and 1991. They are Papers [24], [25], [59], and [66]. 


\section{LIST OF PUBLICATIONS ISSUED UNDER DOE GRANT DE-FG02-84ER40158}

Final Report of the Study of the Theory of High Energy Collision Processes (1969-1984) by Principal Investigator T. T. Wu of Harvard University Supported by DOE

1. The Theory of High Energy Collision Processes (T. T. Wu), U.S. Department of Energy Contract No. DE-AC02-76ER03227, Final Report Number COO-3227-78, for period from June 1, 1969 through May 31, 1984, dated May 31, 1984.

Books Co-authored by Principal Investigator T. T. Wu (1984-2007) Supported by DOE

2. Expanding Protons: Scattering at High Energies (H. Cheng and T. T. Wu), M.I.T. Press, 1987.

3. The Ubiquitous Photon: Helicity Method for QED and QCD (R. Gastmans and T. T. Wu), Oxford University Press, 1990.

Chronological List of Published Papers (1984-2007) Supported by DOE

4. Monopole-Fermion and Dyon-Fermion Bound States. I. General Properties and Numerical Results (P. Osland and T. T. Wu), Nucl. Phys. B 247, 421 (1984).

5. Monopole-Fermion and Dyon-Fermion Bound States. II. Weakly Bound States for the Lowest Angular Momentum (P. Osland and T. T. Wu), Nucl. Phys. B 247, 450 (1984).

6. Fermi Pseudopotentials and Resonances in Arrays (T. T. Wu), pp. 293-306 in Resonances-Models and Phenomena: Proceedings, Bielefeld 1984, S. Albeverio, L. S. Ferreira, and L. Streit, Eds., Springer-Verlag (1984).

7. $p p$ and $\bar{p} p$ Elastic Scattering at $\sqrt{s}=0.55$ to $40 \mathrm{TeV}$ (C. Bourrely, J. Soffer, and T. T. Wu), Phys. Rev. Letts. 54, 757 (1985).

8. Classical Models of Confinement II (H. Lehmann and T. T. Wu), Commun. Math. Phys. 97, 161 (1985).

9. Electromagnetic Missiles (T. T. Wu), J. Appl. Phys. 57, 2370 (1985).

10. Spreading of Electromagnetic Pulses (T. T. Wu and H. Lehmann), J. Appl. Phys. 58, 2064 (1985).

11. Monopole-Fermion and Dyon-Fermion Bound States. III. Monopole-Fermion System with $j=|q|-\frac{1}{2}$ and Large $\kappa|q|$ (P. Osland and T. T. Wu), Nucl. Phys. B 256, 13 (1985).

12. Monopole-Fermion and Dyon-Fermion Bound States. IV. Dyon-Fermion System with $j=|q|-\frac{1}{2}$ and Large $\kappa|q|$ (P. Osland and T. T. Wu), Nucl. Phys. B 256, 32 (1985). 
13. Monopole-Fermion and Dyon-Fermion Bound States. V. Weakly Bound States for the Monopole-Fermion System (P. Osland, C. L. Schultz, and T. T. Wu), Nucl. Phys. B 256, 449 (1985).

14. Monopole-Fermion and Dyon-Fermion Bound States. VI. Weakly Bound States for the Dyon-Fermion System (P. Osland and T. T. Wu), Nucl. Phys. B 261, 687 (1985).

15. Multiple Bremsstrahlung in Gauge Theories at High Energies. V. The Process $e^{+} e^{-} \rightarrow \mu^{+} \mu^{-} \gamma \gamma$ (F. A. Berends, P. DeCausmaecker, R. Gastmans, R. Kleiss, W. Troost, and T. T. Wu), Nucl. Phys. B 264, 243 (1986).

16. Multiple Bremsstrahlung in Gauge Theories at High Energies. VI. The Process $e^{+} e^{-} \rightarrow e^{+} e^{-} \gamma \gamma$ (F. A. Berends, P. DeCausmaecker, R. Gastmans, R. Kleiss, W. Troost, and T. T. Wu), Nucl. Phys. B 264, 265 (1986).

17. Classical Models of Confinement with Moving Charges (H. Lehmann and T. T. Wu), Nucl. Phys. B 271, 166 (1986).

18. A Viable Axion Model (R. D. Peccei, T. T. Wu, and T. Yanagida), Phys. Letts. B 172, 435 (1986).

19. Kazama-Yang Monopole-Fermion Scattering States (K. Olaussen, H. A. Olsen, P. Osland, and I. Øverbø), Nucl. Phys. B 267, 1 (1986).

20. Radiative Capture of Spin- $\frac{1}{2}$ Particles by Magnetic Monopoles (K. Olaussen, H. A. Olsen, P. Osland, and I. Øverbø), Nucl. Phys. B 267, 25 (1986).

21. Perturbative Versus Condensate Effects in Super-Heavy Quarkonia (K. Olaussen and P. Osland), Phys. Letts. B 175, 353 (1986).

22. Cross-Sections for Gluon + Gluon $\rightarrow$ Heavy Quarkonium + Gluon (R. Gastmans, W. Troost, and T. T. Wu), Phys. Letts. B 184, 257 (1987).

23. Caustics in a Simple SU(2) Lattice Gauge Theory Model (J. Bartels and T. T. Wu), Zeitschrift f. Physik C-Particles and Fields 33, 583 (1987).

24. High-Energy, Large-Momentum-Transfer Processes: Ladder Diagrams in $\varphi^{3}$ Theory I (P. Osland and T. T. Wu), Nucl. Phys. B 288, 77 (1987).

25. High-Energy, Large-Momentum-Transfer Processes: Ladder Diagrams in $\varphi^{3}$ Theory II (P. Osland and T. T. Wu), Nucl. Phys. B 288, 95 (1987).

26. Production of Heavy Quarkonia from Gluons (R. Gastmans, W. Troost, and T. T. Wu), Nucl. Phys. B 291, 731 (1987).

27. A Class of Potentials with Extremely Narrow Resonances (A. Grossmann and T. T. Wu), Chinese Jour. Phys. 25, 129 (1987).

28. Impact Picture in the Coulomb Interference Region at High Energies (C. Bourrely, J. Soffer, and T. T. Wu), Phys. Letts. B 196, 237 (1987).

29. Quantum Approach to Beamstrahlung (M. Jacob and T. T. Wu), Phys. Letts. B 197, 253 (1987). 
30. Complex Moments of Parton Distributions and New Sum Rules (C. Bourrely, J. Soffer, and T. T. Wu), Phys. Rev. Letts. 59, 2009 (1987).

31. Impact Picture Predictions for $\bar{p} p$ and $p p$ Elastic Scattering at CERN Collider, FNAL Collider, LHC and SSC (C. Bourrely, J. Soffer, and T. T. Wu), Zeitschrift f. Physik C-Particles and Fields 37, 369 (1988).

32. Quantum Calculation of Beamstrahlung: The Spinless Case (M. Jacob and T. T. Wu), Nucl. Phys. B 303, 373 (1988).

33. Heavy Quarkonia Production from Gluons (R. Gastmans, W. Troost, and T. T. Wu), Nucl. Phys. B (Proc. Suppl.) 1B, 259 (1988).

34. Semiclassical Analysis of the Weak-Coupling Limit of SU(2) Lattice Gauge Theory: The Subspace of Constant Fields (J. Bartels and T. T. Wu), Phys. Rev. D 37, 2307 (1988).

35. Quantum Calculation of Beamstrahlung: The Dirac Case (M. Jacob and T. T. Wu), Nucl. Phys. B 303, 389 (1988).

36. Relativistic Theory of the Decay of Upsilon into Higgs Boson + Photon (G. Fäldt, P. Osland, and T. T. Wu), Phys. Rev. D 38, 164 (1988).

37. Beamstrahlung with Fluctuating Charge Density (M. Jacob and T. T. Wu), Nucl. Phys. B 314, 334 (1989).

38. Beamstrahlung for a Longitudinally Non-Uniform Bunch (M. Jacob and T. T. Wu), Nucl. Phys. B 318, 53 (1989).

39. Beamstrahlung in High Energy Electron-Positron Linear Colliders with Non-Uniform Bunches (M. Jacob and T. T. Wu), Phys. Letts. B 216, 442 (1989).

40. Pair Production in Bunch Crossing (M. Jacob and T. T. Wu), Phys. Letts. B 221, 203 (1989).

41. The Decay $Z \rightarrow \pi^{0} \gamma($ M. Jacob and T. T. Wu), Phys. Letts. B 232, 529 (1989).

42. Coherent Pair Production in Linear Colliders (M. Jacob and T. T. Wu), Nucl. Phys. B 327, 285 (1989).

43. Beamstrahlung in the Deep Quantum Regime (M. Jacob and T. T. Wu), Particle World 1, 20 (1989).

44. Cosmological Implications of Unstable Technibaryons (S. Dodelson), Phys. Rev. D 40, 3252 (1989).

45. Comment on 'Reheated Universe by Unstable Neutrinos' (J. Bernstein and S. Dodelson), Phys. Rev. Letts. 62, 1804 (1989).

46. String Structures on Loop Bundles (R. Coquereaux and K. Pilch), Comm. in Math. Phys. 120, 353 (1989).

47. Noncommutative Geometry and Theoretical Physics (R. Coquereaux), J. Geom. and Phys. 6, No. 1 (1989).

48. Remarks on the Differential Envelopes of Associative Algebras (R. Coquereaux and D. Kastler), Pac. Jour. Math. 137, No. 2 (1989). 
49. Aspects of the Zel'dovich-Sunyaev Mechanism (J. Bernstein and S. Dodelson), Phys. Rev. D 41, 354 (1990).

50. Completely Solvable Models of the Nonlinear Boltzmann Equation, I. Case of Three Velocities (P. Osland and T. T. Wu), J. Math. Phys. 31, 3003 (1990).

51. Baryogenesis in a Baryon-Symmetric Universe (S. Dodelson and L. M. Widrow), Phys. Rev. D 42, 326 (1990).

52. On the Existence of Bound States for a Massive Spin-One Particle and a Magnetic Monopole (H. A. Olsen, P. Osland, and T. T. Wu), Phys. Rev. D 42, 665 (1990).

53. Semiclassical Analysis of the Weak Coupling Limit of SU(2) Lattice Gauge Theory: The Extreme Infrared Region (J. Bartels, B. Raabe, and T. T. Wu), Phys. Rev. D 42, 1233 (1990).

54. $N=4$ Supersymmetric Quantum Mechanics and its Large Order Behaviour (J. J. M. Verbaarschot, P. West, and T. T. Wu), Phys. Letts. B 240, 401 (1990).

55. Large-Order Behaviour of the Supersymmetric Anharmonic Oscillator (J. J. M. Verbaarschot, P. West, and T. T. Wu), Phys. Rev. D 42, 1276 (1990).

56. Experimental Confirmation of the Impact-Picture Predictions for $\bar{p} p$ Elastic Scattering at FNAL Collider (C. Bourrely, J. Soffer, and T. T. Wu), Phys. Letts. B 252, 287 (1990).

57. Baryon-Symmetric Baryogenesis (S. Dodelson and L. M. Widrow), Phys. Rev. Letts. 64, 340 (1990).

58. The Theory of Kaluza-Klein-Jordan-Thiry Revisited (R. Coquereaux and G. Esposito-Farèse), Ann. Inst. Henri Poincaré 52, 113 (1990).

59. High-Energy, Large-Momentum-Transfer Processes: The N-Rung Ladder Diagram of $\varphi^{3}$ Theory (C. Newton and T. T. Wu), J. Math. Phys. 32, 1619 (1991).

60. Proton-Proton and Proton-Antiproton Elastic Scattering at High Energies-Theory, Phenomenology, and Experiment (T. T. Wu), pp. 314-324 in Beyond the Standard Model II, K. Milton, R. Kantowski, and M. A. Samuel, Eds., Proceedings of the International Conference on High Energy Physics, Norman, OK, 1-3 November 1990, World Scientific Publ. Co., Singapore (1991).

61. Real Part of the $\bar{p} p$ Elastic Scattering Amplitude at $\sqrt{s}=546 \mathrm{GeV}$ (C. Bourrely, J. Soffer, and T. T. Wu), Mod. Phys. Letts. A 6, 2973 (1991).

62. Neutrino-Two Photon Vertex (S. Dodelson and G. Feinberg), Phys. Rev. D 43, 913 (1991).

63. Relativistic Bose Gas (J. Bernstein and S. Dodelson), Phys. Rev. Letts. 66, 683 (1991).

64. Phase Structure and the Effective Potential at Fixed Charge (K. M. Benson, J. Bernstein, and S. Dodelson), Phys. Rev. D 44, 2480 (1991).

65. The Pseudodipole as the Limit of a Resonant Cylindrical Dipole (D. K. Freeman), J. Math. Phys. 32, 1961 (1991). 
66. The Asymptotics of $\phi^{3}$ Ladder Diagrams in Six Dimensions: High Energy and Large Momentum Transfer (R. Gastmans, W. Troost, and T. T. Wu), Nucl. Phys. B 365, 404 (1991).

67. Excess of $\tau^{+} \tau^{-} V$ Events in $Z$ Decays: A Naive Look (R. Gastmans, W. Troost, and T. T. Wu), Phys. Letts. B 271, 425 (1991).

68. Radiated Energy Loss Due to Beamstrahlung in High-Energy Electron-Positron Linear Colliders (M. Jacob, T. T. Wu, and G. Zobernig), Zeitschrift f. Physik C-Particles and Fields 53, 479 (1992).

69. Macroscopic Lack of Localization in Quantum Radiative Processes (M. Jacob and T. T. Wu), Phys. Letts. A 162, 304 (1992).

70. Multiphoton Radiation in Quantum Beamstrahlung (M. Jacob and T. T. Wu), Nucl. Phys. B 371, 59 (1992).

71. Stability of Three Unit-Charge Systems (A. Martin, J.-M. Richard, and T. T. Wu), Phys. Rev. A 46, 3697 (1992).

72. Generalization of the Pseudopotential to an Interaction with Nonzero Range (D. K. Freeman), J. Math. Phys. 33, 2215 (1992).

73. Beamstrahlung: A Status Report with Recent Results (M. Jacob and T. T. Wu), pp. 60-63 in Proceedings of the 1991 Joint International Lepton-Photon Symposium and Europhysics Conference on High Energy Physics, Geneva, Switzerland, July 25-August 1, 1991, S. Hegarty, K. Potter, and E. Quercigh, Eds., World Scientific Publ. Co., Singapore (1992).

74. Parameters in the Electroweak Theory, I. Point Splitting as Regularization (P. Osland and T. T. Wu), Zeitschrift f. Physik C-Particles and Fields 55, 569 (1992).

75. Parameters in the Electroweak Theory, II. Quadratic Divergences (P. Osland and T. T. Wu), Zeitschrift f. Physik C-Particles and Fields 55, 585 (1992).

76. Parameters in the Electroweak Theory, III. Masses of the Top Quark and the Higgs Boson (P. Osland and T. T. Wu), Zeitschrift f. Physik C—Particles and Fields 55, 593 (1992).

77. Masses of the Higgs Boson and the Top Quark in the Standard Model from Absence of Divergences (P. Osland and T. T. Wu), Phys. Letts. B 291, 315 (1992).

78. Baryogenesis, Dark Matter, and the Width of the $Z$ (S. Dodelson, B. R. Greene, and L. M. Widrow), Nucl. Phys. B 372, 467 (1992).

79. Reionization and Decaying Dark Matter (S. Dodelson and J. M. Jubas), Phys. Rev. D 45, 1076 (1992).

80. Transverse Distribution Effects in Beamstrahlung (M. Jacob and T. T. Wu), Zeitschrift f. Physik C-Particles and Fields 58, 279 (1993).

81. Optimized Lower Bounds in the Three-Body Problem (J.-L. Basdevant, A. Martin, J.-M. Richard, and T. T. Wu), Nucl. Phys. B 393, 111 (1993).

82. Predictions for the Masses of the Higgs Boson and the Top Quark (P. Osland and T. T. Wu), Int. J. Mod. Phys. A (Proc. Suppl.) 3B, 131 (1993). 
83. Comparison of Theoretical Predictions from the Impact Picture with the Recent UA4 Data (C. Bourrely, J. Soffer, and T. T. Wu), Phys. Letts. B 315, 195 (1993).

84. Can the Higgs Boson Be Detected at the Fermilab Tevatron Collider? (C. Newton, P. Osland, and T. T. Wu), Zeitschrift f. Physik C-Particles and Fields 61, 421 (1994).

85. Point-Splitting Regularization for Gauge Theories: Quantum Electrodynamics (C. Newton, P. Osland, and T. T. Wu), Zeitschrift f. Physik C-Particles and Fields 61, 441 (1994).

86. Mass Relations in the Two Higgs Doublet Model from the Absence of Quadratic Divergences (C. Newton and T. T. Wu), Zeitschrift f. Physik C-Particles and Fields 62, 253 (1994).

87. Resonances and Stability in Arrays of Pseudodipoles (D. K. Freeman), J. Electrom. Waves \& Applic. 8, 537 (1994).

88. Expanding Protons Seen by HERA (C. Bourrely, J. Soffer, and T. T. Wu), Phys. Letts. B 339, 322 (1994).

89. Generalized Gauge Transformation: Abelian Case (R. Gastmans, C. Newton, and T. T. Wu), Phys. Letts. B 341, 84 (1994).

90. Variational-Principle Formulation of the Two-Term Theory for Arrays of Cylindrical Dipoles (D. K. Freeman and T. T. Wu), IEEE Trans. Antennas Propagat. 43, 340 (1995).

91. Behavior of Total and Elastic Cross Sections at Very High Energies (T. T. Wu), pp. 291-302 in Proceedings of the Workshop on Quantum Infrared Physics, H. M. Fried and B. Müller, Eds., World Scientific Publ. Co., Singapore (1995).

92. Mass Relations in the Standard Model: The Higgs Boson and the Top Quark Masses," (C. Newton, P. Osland, and T. T. Wu), pp. 351-360 in Quantum Systems: New Trends and Methods, Proceedings of the International Workshop, Minsk, Republic of Belarus, May 23-29, 1994, A. O. Barut, I. D. Feranchuk, Ya. M. Shnir, and L. M. Tomil'chik, Eds., World Scientific Publ. Co., Singapore (1995).

93. Stability of Systems of Three Arbitrary Charges: General Properties (A. Martin, J.-M. Richard, and T. T. Wu), Phys. Rev. A 52, 2557 (1995).

94. Scattering by Spheres at Short Wavelengths - from Maxwell to HERA (T. T. Wu), pp. 45-54, in Annales des Journées Maxwell, Proceedings of the Journées Maxwell '95, 6-9 June 1995, Bordeaux-Lac, France, J.-F. Eloy, Ed., CESTA, Le Barp, France (1995).

95. Quarter Century of Rising Total Cross Sections (C. Bourrely, J. Soffer, and T. T. $\mathrm{Wu}$, pp. 15-22 in Frontiers in Strong Interactions (Proceedings of the VIth International Conference on Elastic and Diffractive Scattering, 20-24 June 1995, Blois, France), P. Chiappetta, M. Hauguenauer, and J. Tran Thanh Van, Eds., Editiones Frontières, Blois, France (1996).

96. Generalized Gauge Transformations: SU(2) Yang-Mills Case (R. Gastmans, C. Newton, and T. T. Wu), Phys. Rev. D 54, 5302 (1996). 
97. A New Variant of Symmetry Breaking for Quark Mass Matrices (H. Lehmann, C. Newton, and T. T. Wu), Phys. Letts. B 384, 249 (1996).

98. On Bose-Einstein Condensation, pp. 76-81 in Quantum Chromodynamics: Collisions, Confinement, and Chaos, H. M. Fried and B. Müller, Eds., World Scientific Publ. Co., Singapore (1997).

99. New Singularities in Non-Relativistic Coupled Channel Scattering, I. Second Order (N. N. Khuri and T. T. Wu), Phys. Rev. D 56, 6779 (1997).

100. New Singularities in Non-Relativistic Coupled Channel Scattering, II. Fourth Order (N. N. Khuri and T. T. Wu), Phys. Rev. D 56, 6785 (1997).

101. Generalized Gauge Transformations: Pure Yang-Mills Case (R. Gastmans and T. T. Wu), Phys. Rev. D 57, 1203 (1998).

102. Bose-Einstein condensation in an external potential at zero temperature: General theory (T. T. Wu), Phys. Rev. A 58, 1465 (1998).

103. Universality of Low-Energy Scattering in $(2+1)$ Dimensions (K. Chadan, N. N. Khuri, A. Martin, and T. T. Wu), Phys. Rev. D 58(2), 025014 (1998).

104. Optimized Lower Bound on Four-Body Hamiltonians (A. Benslama, A. Metatla, A. Bachkhaznadji, S. R. Zouzou, A. Krikeb, J.-L. Basdevant, J.-M. Richard, and T. T. Wu), Few-Body Systems 24, 39 (1998).

105. Bose-Einstein Condensation in an External Potential at Zero Temperature: Solitary-Wave Theory (D. Margetis), J. Math. Phys. 40, 5522 (1999).

106. $\gamma \gamma$ Total Cross-Section at High Energies (C. Bourrely, J. Soffer, and T. T. Wu), Mod. Phys. Letts. A 15(1), 9 (2000).

107. Solar Mikheyev-Smirnov-Wolfenstein Effect with Three Generations of Neutrinos (P. Osland and T. T. Wu), Phys. Rev. D 62(1), 013008 (2000).

108. Stability Domain of Systems of Three Arbitrary Charges (A. Krikeb, A. Martin, J.-M. Richard, and T. T. Wu), Few-Body Systems 29, 237 (2000).

109. Collective Effects from Induced Behavior (I. O. Stamatescu and T. T. Wu), The European Physical Journal B 16(4), 741 (2000).

110. Universal Quark-Lepton Mixing and Determination of Neutrino Masses (P. Osland and T. T. Wu), presented at XXXth Internation Conference on High Energy Physics, Osaka, Japan, 27 July-2 August, 2000; also arXiv:hep-ph/0006185v1 16Jun2000.

111. Mikheyev-Smirnov-Wolfenstein Effect for Linear Electron Density (H. Lehmann, P. Osland, and T. T. Wu), Communications in Mathematical Physics 219(1), 77 (2001).

112. Three-Neutrino MSW Effect and the LNW Mass Matrix (P. Osland and T. T. Wu), Physica Scripta T93, 37 (2001).

113. Scattering and Production Processes at High Energies (T. T. Wu), Chapter 4.3.4 in Scattering-Scattering and Inverse Scattering in Pure and Applied Science, Vol. 2, R. Pike and P. Sabatier, Eds., pp. 1582-1604, Academic Press, London (2002). 
114. Bound States in $n$ Dimensions (Especially $n=1$ and $n=2$ ) (N. N. Khuri, A. Martin, and T. T. Wu), Few-Body Systems 31, 83 (2002).

115. Bound States in One and Two Spatial Dimensions (K. Chadan, N. N. Khuri, A. Martin, and T. T. Wu), J. Math. Phys. 44(2), 406 (2003).

116. Tsunami Run-Up and Draw-Down on a Plane Beach (G. F. Carrier, T. T. Wu, and H. Yeh), J. Fluid Mech. 475, 79 (2003).

117. Yang-Mills Theory for Non-semisimple Groups (J. Nuyts and T. T. Wu), Phys. Rev. D $67(2), 025014$ (2003).

118. Neutrino Masses From Universal Fermion Mixing (R. Gastmans, P. Osland, and T. T. Wu), Phys. Rev. D 67(5), 053005 (2003).

119. Impact-Picture Phenomenology for $\pi^{ \pm} p, K^{ \pm} p$ and $p p, \bar{p} p$ Elastic Scattering at High Energies (C. Bourrely, J. Soffer, and T. T. Wu), European Physical Journal C 28(1), 97 (2003).

120. Remarks on Yang-Mills Theory (T. T. Wu), in Symmetry $\&$ Modern Physics: Yang Retirement Symposium, A. Goldhaber, R. Shrock, J. Smith, G. Sterman, P. van Nieuwenhuizen, W. Weisberger, Eds., World Scientific, Singapore, pp. 199-204 (2003).

121. Bound States in Two Spatial Dimensions in the Noncentral Case (A. Martin and T. T. Wu), J. Math. Phys. 45(3), 922 (2004).

122. Universality of Low-Energy Scattering in $2+1$ Dimensions: The Nonsymmetric Case (N. N. Khuri, A. Martin, P. C. Sabatier, and T. T. Wu), J. Math. Phys. 46, 032103 (2005).

123. Why the Real Part of the Proton-Proton Scattering Amplitude Should be Measured at the LHC (C. Bourrely, N. N. Khuri, A. Martin, J. Soffer, and T. T. Wu), pp. 41-44 in Proceedings of the XIth International Conference on Elastic and Diffractive Scattering: Toward High Energy Frontiers, Château de Blois, France, May 15-20, M. Haguenauer, B. Nicolescu, and J. Tran Thanh Van, Eds., The Gioi Publishers, Vietnam (2006).

124. Why the Real Part of the Proton-Proton Scattering Amplitude Should be Measured at the LHC (C. Bourrely, N. N. Khuri, A. Martin, J. Soffer, and T. T. Wu), p. 125 in V. N. Gribov Memorial Volume-Quarks, Hadrons, and Strong Interactions, Yu. L. Dokshitzer, P. Levai, and J. Nyiri, Eds., World Scientific Publishing Co., Singapore (2006).

125. Impact Picture for the Analyzing Power $A_{N}$ in Very Forward $p p$ Elastic Scattering (C. Bourrely, J. Soffer, and T. T. Wu), Phys. Rev. D 76, 053002 (2007). 